\title{
Impact of smoking, alcohol consumption, and NSAID use on risk for and phenotypes of eosinophilic esophagitis
}

\author{
N. T. Koutlas, ${ }^{1}$ S. Eluri,,${ }^{1,2}$ S. Rusin, ${ }^{3}$ I. Perjar, ${ }^{3}$ J. Hollyfield,${ }^{3}$ J. T. Woosley, ${ }^{3}$ N. J Shaheen, ${ }^{1,2}$ E. S Dellon ${ }^{1,2}$ \\ ${ }^{1}$ Center for Esophageal Diseases and Swallowing, ${ }^{2}$ Center for Gastrointestinal Biology and Disease, Division of Gas- \\ troenterology and Hepatology, Department of Medicine, and ${ }^{3}$ Department of Pathology and Laboratory Medicine, \\ University of North Carolina School of Medicine, Chapel Hill, North Carolina, USA
}

SUMMARY. There are few data exploring modifiable risk factors for eosinophilic esophagitis (EoE). We aimed to determine if smoking, alcohol consumption, and nonsteroidal anti-inflammatory drug (NSAID) use were risk factors for EoE, and to assess their impact on EoE phenotypes and treatment outcomes. We performed a case-control study analyzing data collected from a prospective cohort of adults undergoing upper endoscopy for symptoms of esophageal dysfunction. Incident EoE cases were diagnosed via consensus guidelines. Exposure data were collected via standardized patient questionnaire. Follow-up assessments for cases were made after treatment, with histologic response defined as $<15$ eosinophils per high-power field (eos/hpf). Exposures were compared between EoE cases and controls, among EoE cases with and without fibrostenosis, and among EoE responders and nonresponders. A total of 115 cases and 225 controls were analyzed. Cases were less likely to have ever smoked cigarettes $(23 \%$ vs. $47 \%, P<0.001)$ or currently use NSAIDs $(17 \%$ vs. $40 \%, P<0.001)$ compared to controls. These relations persisted after multivariate analysis. Although alcohol use was more common among cases $(75 \%$ vs. $51 \%, P<0.001)$, the effect was abrogated after multivariate analysis. Smoking, alcohol, and NSAID use were not associated with the fibrostenotic phenotype. There was a trend toward improved histologic response among EoE patients concomitantly using NSAIDs $(87 \%$ vs. 63\%, $P=0.08$; aOR $6.97(95 \%$ CI: $0.81-60.3)$. In conclusion, NSAID and smoking were inversely associated with EoE compared to endoscopy-based controls. Alcohol use was more prevalent in the EoE cases, although not an independent risk factor. Concomitant NSAID use may improve treatment response and is worthy of future study.

KEY WORDS: eosinophilic esophagitis, exposures, habits, medications, risk factors.

\section{INTRODUCTION}

Eosinophilic esophagitis (EoE) is a chronic immune/allergen-mediated disease characterized by symptoms of esophageal dysfunction and eosinophilic inflammation of the esophageal epithelium. ${ }^{1,2} \mathrm{EoE}$ was rarely recognized before the 1990s, but is now

Address correspondence to: Dr. Evan S. Dellon MD, MPH, CB\#7080, Bioinformatics Building, 130 Mason Farm Rd.

UNC-CH, Chapel Hill, NC 27599-7080, USA. Email: edellon@med.unc.edu

Conflicts of interest: The authors declare that they have no conflict of interest.

Specific author contributions: Project conception: Nathaniel T. Koutlas, Evan S. Dellon; data analysis/interpretation: Nathaniel T. Koutlas, Evan S. Dellon; drafting of the article: Nathaniel T. Koutlas, Evan S. Dellon; critical revision: Nathaniel T. Koutlas, Swathi Eluri, Spencer Rusin, Irina Perjar, Johnathan Hollyfield, John T. Woosley, Nicholas J. Shaheen, Evan S. Dellon; approved final draft: Nathaniel T. Koutlas, Swathi Eluri, Spencer Rusin, Irina Perjar, Johnathan Hollyfield, John T. Woosley, Nicholas J. Shaheen, Evan S. Dellon. considered a significant cause of upper GI morbidity in both children and adults. ${ }^{3,4}$ More than half of patients presenting to the emergency department with a food impaction are later diagnosed with EoE. ${ }^{5-7}$ The current estimated prevalence of EoE in the United States is 56.7 cases per 100,000 people and it is most commonly found in Caucasian male adults under the age of $50 .^{8-10}$ Despite the increasing prevalence and recognition, little is known about risk factors that predispose to EoE, including modifiable behaviors and habits.

Smoking cigarettes, consuming alcohol, and taking nonsteroidal anti-inflammatory drugs (NSAIDs) are highly common practices in the United States, with known associations with esophageal and upper GI disorders. ${ }^{11-17}$ Alcohol and smoking have been shown to be risk factors for gastroesophageal reflux disease (GERD) and esophageal squamous cell carcinoma and may exacerbate erosive esophagitis and 
esophageal strictures. ${ }^{1-15}$ In contrast, NSAIDs may offer some protection from Barrett's esophagus and both histologic types of esophageal cancer. ${ }^{16,17}$ These factors, however, have not been extensively investigated as risk or mitigating factors in $\mathrm{EoE},{ }^{11}$ and the impact of these habits and medications on the presentation and outcomes of EoE is unknown.

The aim of this study is to determine if smoking, alcohol consumption, and NSAID use were risk factors for EoE, and to assess the impact of these factors on EoE phenotypes and treatment outcomes. Based on associations with other upper GI pathology, we hypothesized that alcohol consumption and cigarette smoking would be more prevalent among EoE cases in contrast to non-EoE controls, while NSAID use would be more common in the controls. We also predicted that patients with exposure to alcohol and cigarette smoking would be more likely to have a fibrostenotic EoE phenotype as defined by esophageal narrowing, strictures, or need for esophageal dilation, and would be less likely to have histologic response to treatment.

\section{MATERIALS AND METHODS}

\section{Study design and case definition}

We conducted a case-control study nested within data collected from a prospective cohort study of incident EoE cases and non-EoE controls. ${ }^{18-21}$ The study population was composed of adult patients who underwent an outpatient upper endoscopy at the University of North Carolina (UNC) from 2011 to 2015. Patients who underwent a clinically indicated upper endoscopy were recruited if they were 18 years of age or older and had symptoms of esophageal dysfunction (i.e. dysphagia, food impaction, heartburn, or chest pain). Criteria for exclusion included known diagnosis of EoE or other eosinophilic GI disorders, known esophageal cancer, known esophageal varices, previous esophageal surgery, active anticoagulation, medical instability or comorbidities precluding enrollment in the opinion of the endoscopist, and inability to read or understand the consent form. Subjects provided informed consent and were enrolled prior to the endoscopy. The UNC Institutional Review Board approved this study.

Cases of EoE were diagnosed per consensus guidelines. ${ }^{1,2}$ Specifically, EoE cases were required to have a typical symptom of esophageal dysfunction, an esophageal biopsy with at least 15 eosinophils per high power field (eos/hpf) after an 8-week trial of any available PPI agent (20-40 mg twice daily, prescribed at the discretion of the clinician), and all other causes of esophageal eosinophilia excluded. Non-EoE controls were patients who did not meet these criteria. This specific control group was designed to represent a group of patients with suspected EoE (i.e. patients undergoing upper endoscopy for evaluation of symptoms of esophageal dysfunction) but could represent any number of eventual diagnoses. Of note, patients with proton pump inhibitor-responsive esophageal eosinophilia (PPI-REE) were not included in the present analysis.

\section{Clinical data, exposures, and outcomes}

All data on age, demographics, clinical symptoms, comorbidities including atopic diseases, endoscopic features, and histologic findings were collected using standardized case report forms. Exposure data were ascertained via a prospectively administered patient questionnaire, which was administered prior to endoscopy and prior to case/control determination. Subjects were asked whether they had ever smoked cigarettes, used chewing tobacco, or consumed alcohol. If yes, subjects were then asked if they were current users and to quantify their use. For alcohol, one drink was defined as either $1 \mathrm{oz}$ of hard alcohol, $4 \mathrm{oz}$ of wine, or $12 \mathrm{oz}$ of beer. For NSAIDs, subjects were asked if they were current users. Specific NSAID medications, doses, and durations were not recorded, as these details were not included in the original questionnaire.

During endoscopy, research biopsies were obtained from the proximal, mid, and distal esophagus to maximize sensitivity for EoE diagnosis. ${ }^{15}$ Gastric and duodenal research biopsies were also collected to exclude eosinophilic gastroenteritis. Clinical biopsies were taken at the discretion of the endoscopist. After biopsy specimen collection, the study pathologists quantified the esophageal eosinophil count using previously validated methods. ${ }^{22,23}$ To summarize, slides were masked to case/control status, digitized, and reviewed with Aperio ImageScope (Aperio Technologies, Vista, CA). Pathologists did not have access to endoscopy reports or clinical findings. Five microscopic fields were examined from each of the research biopsies to determine the maximum eosinophil density (eosinophils $/ \mathrm{mm}^{2}$ ). Maximum eosinophil densities were converted to eosinophil counts (eos/hpf) to compare the results to prior studies by using the hpf size of $0.24 \mathrm{~mm}^{2}$, which is the most commonly used field size in the literature. ${ }^{24}$ Other histologic findings associated with EoE were recorded, such as eosinophilic degranulation, eosinophilic microabscesses (cluster of $\geq 4$ eos), basal zone hyperplasia, spongiosis, and lamina propria fibrosis (only if adequate subepithelial stroma was present).

EoE cases were treated at the clinical discretion of their gastroenterologist. At UNC, first-line treatment options included swallowed/topical corticosteroids (either a viscous budesonide suspension at $2 \mathrm{mg} /$ day or fluticasone multidose inhaler at 1760 $\mathrm{mcg} / \mathrm{d}$ ) or dietary elimination (six-food elimination diet). ${ }^{25}$ After 8 weeks of clinically indicated treatment 
Table 1 Patient characteristics

\begin{tabular}{lccr}
\hline & Non-EoE controls $(n=225)$ & EoE cases $(n=115)$ & $p^{*}$ \\
\hline Age at diagnosis (mean \pm SD) & & $38.0 \pm 12.6$ & $<0.001$ \\
Male $(n, \%)$ & $53.8 \pm 14.1$ & $67(58)$ & $<0.001$ \\
White $(n, \%)$ & $82(36)$ & $109(95)$ & 0.001 \\
Symptoms $(n, \%)$ & $182(81)$ & $113(98)$ & $<0.001$ \\
Dysphagia & $159(71)$ & $10(9)$ & $<0.001$ \\
Heartburn & $83(37)$ & $8(7)$ & 0.15 \\
Abdominal pain & $27(12)$ & $1(1)$ & 0.14 \\
Nausea/vomiting & $8(4)$ & $74(76)$ & 0.06 \\
Any atopic disorder $(n, \%)$ & $142(66)$ & $36(32)$ & 0.84 \\
Asthma & $68(31)$ & $11(10)$ & 0.37 \\
Atopic dermatitis & $22(10)$ & $77(69)$ & 0.02 \\
Allergic rhinitis/sinusitis & $124(56)$ & $43(38)$ & 0.001 \\
Food allergies & $46(21)$ & $4(3)$ & $<0.001$ \\
EGD findings $(n, \%)$ & & $97(84)$ & $<0.001$ \\
Normal & $42(19)$ & $36(31)$ & 0.002 \\
Rings & $32(14)$ & $36(31)$ & $<0.001$ \\
Strictures & $38(17)$ & $7(6)$ & 0.005 \\
Narrowing & $10(4)$ & $101(88)$ & $<0.001$ \\
Crepe paper mucosa & $2(1)$ & $57(50)$ & $<0.001$ \\
Furrows & $15(7)$ & $52(45)$ & $<0.001$ \\
White plaques/exudates & $11(5)$ & $15(13)$ & $<0.001$ \\
Edema/decreased vascularity & $6(3)$ & $35(30)$ & 0.97 \\
Hiatal hernia & $92(41)$ & $119.6 \pm 113.0$ & $<0.001$ \\
Dilation performed & $68(30)$ & & \\
Baseline max eosinophil count (mean \pm SD) & $3.2 \pm 10.0$ & & \\
\hline
\end{tabular}

*means compared with t-test; proportions compared with chi-square.

with topical steroids or 6 weeks of treatment with dietary elimination, patients underwent repeat upper endoscopy and biopsy, with repeat collection of the endoscopic and histologic data noted above. For the purposes of the present analysis, histologic treatment response was defined as a posttreatment biopsy with $<15$ eos/hpf. ${ }^{26}$

\section{Statistical analysis}

Descriptive statistics were used to summarize the demographic, endoscopic, histologic, and exposure data. Bivariate analysis was performed to compare risk factors between EoE cases and non-EoE controls to test our first hypothesis. Means were compared with t-tests and proportions were compared with Chi-square. We also performed multivariate logistic regression controlling for potential confounders (i.e. age, sex, race, education level as a proxy for socioeconomic status, and atopy) to calculate adjusted odd ratios (aORs). To test whether smoking, alcohol, or NSAIDs impacted clinical phenotype, we compared exposures in EoE patients with the endoscopic findings of fibrostenosis (i.e. strictures, esophageal narrowing, or need for esophageal dilation) to those without findings of fibrostenosis. To assess whether smoking, alcohol, or NSAIDs impacted histologic response to treatment, we compared exposures between histologic responders and nonresponders among the EoE cases with follow up data. Multivariate logistic regression was also performed to calculate aORs.

\section{RESULTS}

\section{Baseline characteristics and exposure status}

A total of 115 EoE cases and 225 non-EoE controls were included in this study (Table 1). EoE cases were younger ( 38 vs. 54 years; $P<0.001$ ) and more likely to be male $(58 \%$ vs. $36 \% ; P<0.001)$ than non-EoE controls. Endoscopic findings of rings, strictures, narrowing, furrows, edema, and white plaques were more common in the EoE group, and the baseline peak eosinophil count was markedly elevated in cases compared to controls $(120 \mathrm{eos} / \mathrm{hpf}$ vs. $3 ; P<0.001)$ (Table 1). The most common diagnoses in the control group included GERD (50\%; with $8 \%$ having a peptic stricture), esophageal dysmotility $(17 \%)$, nonreflux- related stricture $(12 \%)$, and functional $(11 \%)$.

EoE cases were less likely to have ever smoked cigarettes compared to the endoscopy-based non-EoE controls ( $23 \%$ vs. $47 \%, P<0.001)$ and to currently use NSAIDs $(17 \%$ vs. $40 \%, P<0.001$ (Table 2 ; Fig. 1$)$. These relations were slightly attenuated, but persisted after multivariate analysis adjusting for potential confounding factors (Table 3). Of note, controls with GERD did not have higher rates of smoking than controls with non-GERD symptoms or diagnoses. The aOR for EoE in NSAID users was 0.36 (95\% CI: $0.17-$ $0.76)$ and the aOR for ever smoking was $0.47(0.24$ 0.92). Current alcohol use was more common among EoE cases $(75 \%$ vs. $51 \%, P<0.001)$ (Table 2), but the effect was abrogated after multivariate analysis (Table 3). 
Table 2 Tobacco, alcohol use, and NSAIDs in EoE cases and controls

\begin{tabular}{lccc}
\hline & Non-EoE controls $(n=225)$ & EoE cases $(n=115)$ & $p^{*}$ \\
\hline Current NSAID $(n, \%)$ & $87(40)$ & $19(17)$ & $<0.001$ \\
Smoking $(n, \%)$ & $105(47)$ & $26(23)$ & $<0.001$ \\
Ever & $25(11)$ & $5(5)$ & 0.04 \\
Current & & & $<0.001$ \\
Number of daily cigarettes & $9(36)$ & $5(20)$ & \\
$1-10$ & $12(48)$ & $0(0)$ & \\
$11-20$ & $4(16)$ & $0(0)$ & 0.25 \\
$21-40$ & $0(0)$ & $0(0)$ & $<0.001$ \\
$>40$ & $19(9)$ & $14(13)$ & \\
Chewing tobacco $(n, \%)$ & $4(2)$ & $5(5)$ & \\
Ever & $108(49)$ & $28(25)$ & \\
Current & $113(51)$ & $82(75)$ & \\
Alcohol $(n, \%)$ & $107(49)$ & $37(34)$ & \\
Previous or never & $53(24)$ & $29(26)$ & \\
Current & $38(18)$ & $29(26)$ & \\
Number of weekly drinks & $16(7)$ & $2(11)$ & \\
$1-2$ & $2(1)$ & $43(39)$ & 0.02 \\
$3-7$ & $56(26)$ &
\end{tabular}

*proportions compared with chi-square.

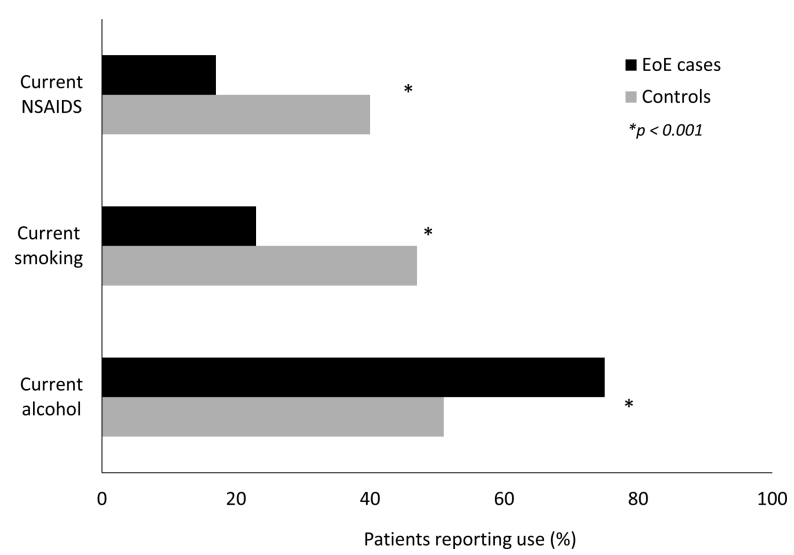

Fig. 1 NSAID, smoking, and alcohol use among EoE cases (black bars) and controls (gray bars). Proportions were compared with chi-square.

\section{Impact of NSAIDs, smoking, and alcohol on fibrostenosis and treatment response}

Rates of NSAID, smoking, and alcohol use were similar among EoE patients with fibrostenosis $(n=60$ with stricture, narrowing or dilation) and without fibrostenosis $(n=55)$ (Table 4$)$. In general, there was no relation between stricture severity or need for dilation (data not shown). However, EoE patients who consumed more than 7 drinks of alcohol per week had more severe (narrow) strictures at baseline compared to those who had 7 or less drinks per week $(7 \pm 3 \mathrm{~mm}$ vs. $11 \pm 3 \mathrm{~mm} ; P=0.03$ ).

A total of $86 \mathrm{EoE}$ cases had complete posttreatment data available for analysis. While histologic response rates were similar for EoE cases with smoking and alcohol exposures, there was a trend for improved histologic response for EoE patients concomitantly using
NSAIDs ( $87 \%$ vs. $63 \%, P=0.08)$ (Table 5), with an aOR of 6.97 (95\% CI: 0.81-60.3; adjusted for age, sex, race, education level, and atopy). Interestingly, the two patients who were currently using chewing tobacco were both histologic nonresponders, but the small number of overall chewing tobacco uses precluded more detailed analysis of this exposure.

\section{DISCUSSION}

A number of factors such as food-based allergens, aeroallergens, and early life exposures such as antibiotic use have been implicated in previous studies as potential triggers for EoE. ${ }^{27}$ However, little is known about the effects of smoking, alcohol, and NSAIDs, on EoE. This is an especially glaring gap in our knowledge, given the important role these risk factors play in other upper gastrointestinal disease processes. In this analysis of a prospectively recruited cohort of EoE cases and endoscopy-based non-EoE controls, we aimed to determine if smoking, alcohol, and/or NSAIDs were risk factors for EoE and if exposure to these substances impacted EoE phenotypes and treatment response. We found an inverse relationship between smoking cigarettes and EoE as well as NSAID use and EoE, and these relationships held after adjusting for potential confounding factors including age, sex, race, atopy, and educational level. While current alcohol consumption was more common in the EoE cases, the association did not persist after multivariate analysis. We did not find a correlation between any of the exposures and presence of a fibrostenotic phenotype. 
Table 3 Odds of EoE based on NSAID, smoking, or alcohol status

\begin{tabular}{lcc}
\hline & Odds ratio $(95 \% \mathrm{CI})$ & Adjusted odds ratio $(95 \% \mathrm{CI})$ \\
\hline Current NSAID use & $0.31(0.17-0.54)$ & $0.36(0.17-0.76)^{\dagger}$ \\
Ever smoked cigarettes & $0.34(0.20-0.57)$ & $0.47(0.24-0.92)^{\ddagger}$ \\
Current alcohol use & $2.80(1.69-4.63)$ & $1.57(0.80-3.08)^{\S}$ \\
Daily alcohol use & $1.83(1.12-2.99)$ & $1.05(0.54-2.03)^{\S}$ \\
\hline
\end{tabular}

${ }^{\dagger}$ when controlling for age, sex, race, education level, and atopy;

${ }^{\ddagger}$ when controlling for age, sex, race, education level, alcohol consumption, and atopy;

$\S$ when controlling for age, sex, race, education level, smoking, and atopy.

Table 4 Relation of tobacco, alcohol, and NSAIDs to fibrostenosis in EoE cases

\begin{tabular}{lcrr}
\hline & \multicolumn{3}{c}{ EoE cases $(n=115)$} \\
\cline { 2 - 3 } & No stricture, narrowing, or dilation $(n=55)$ & Stricture, narrowing, or dilation $(n=60)$ & $p^{*}$ \\
\hline Current NSAID use $(n, \%)$ & $11(21)$ & $8(14)$ & 0.33 \\
Smoking $(n, \%)$ & $13(25)$ & $13(22)$ & 0.79 \\
$\quad$ Ever & $3(6)$ & $2(3)$ & 0.58 \\
$\quad$ Current & $7(13)$ & $7(12)$ & 0.86 \\
Chewing tobacco $(n, \%)$ & $1(2)$ & $4(7)$ & 0.20 \\
$\quad$ Ever & $42(81)$ & $40(69)$ & 0.16 \\
Current & $24(46)$ & $19(33)$ & 0.15 \\
Alcohol $(n, \%)$ & Current & & \\
$>7$ drinks per week & &
\end{tabular}

* proportions compared with chi-square.

Table 5 Impact of tobacco, alcohol, and NSAIDs on treatment response in EoE cases

\begin{tabular}{|c|c|c|c|}
\hline & \multicolumn{2}{|c|}{ EoE cases with follow up data $(n=86)^{\dagger}$} & \multirow[b]{2}{*}{$p^{*}$} \\
\hline & No histologic response $(n=28)$ & Histologic response $(n=58)$ & \\
\hline \multicolumn{4}{|c|}{ NSAID use $(n, \%)$} \\
\hline Not current & $26(37)$ & $45(63)$ & \multirow[t]{2}{*}{0.08} \\
\hline Current & $2(13)$ & $13(87)$ & \\
\hline \multicolumn{4}{|l|}{ Smoking $(n, \%)$} \\
\hline Never & $21(32)$ & $47(68)$ & \multirow[t]{2}{*}{0.60} \\
\hline Ever & $7(39)$ & $11(61)$ & \\
\hline \multicolumn{4}{|c|}{ Chewing tobacco $(n, \%)$} \\
\hline \multicolumn{4}{|c|}{ Ever used } \\
\hline No & $24(32)$ & $53(68)$ & \multirow[t]{2}{*}{0.47} \\
\hline Yes & $4(44)$ & $5(56)$ & \\
\hline \multicolumn{4}{|l|}{ Current use } \\
\hline No & $26(32)$ & $58(68)$ & \multirow[t]{2}{*}{0.05} \\
\hline Yes & $2(100)$ & $0(0)$ & \\
\hline \multicolumn{4}{|l|}{ Alcohol $(n, \%)$} \\
\hline \multicolumn{4}{|l|}{ Current use } \\
\hline No & $9(45)$ & $11(55)$ & \multirow[t]{2}{*}{0.19} \\
\hline Yes & $19(29)$ & $47(71)$ & \\
\hline \multicolumn{4}{|c|}{$>7$ drinks per week } \\
\hline No & $22(40)$ & $34(60)$ & \multirow[t]{2}{*}{0.06} \\
\hline Yes & $6(20)$ & $24(80)$ & \\
\hline
\end{tabular}

${ }^{\dagger}$ nonresponders defined by $\geq 15$ eos/hpf on esophageal biopsy; responders defined by $<15$ eos/hpf on esophageal biopsy;

* proportions compared with chi-square.

While our risk factor analysis is unique in the EoE literature and thus difficult to contextualize, our general lack of association with fibrostenosis is in contrast to another study. Lipka and colleagues assessed $39 \mathrm{EoE}$ patients with fibrostenosis and 25 with an inflammatory phenotype. ${ }^{11}$ Alcohol, smoking, and NSAIDs did not differ between the two phenotypes, but patients with these exposures and a long diagnostic delay ( $>7$ years) presented with more severe strictures than patients without these exposures. Their study differed from ours in its retrospective design and methods for characterizing exposures and fibrostenosis, thus results are not directly comparable. However, upon subanalysis we found that EoE patients who consumed more than 7 drinks per week had more severe strictures, a finding similar to that of 
the Lipka study. It is plausible to think that alcohol or compounds in swallowed cigarette smoke or tobacco could cause some direct toxicity or injury to the esophagus and worsen findings of EoE. However, epithelial barrier impairments have been shown to be prominent in $\mathrm{EoE},{ }^{28-30}$ which is why we had originally hypothesized that smoking would be positively associated with the condition, and not inversely associated as we found. It is not clear if not smoking is a proxy measure for another behavior or exposure that itself might increase the risk of EoE, and this will need to be a direction of future research.

This study also revealed a trend toward improved histologic response to treatment in EoE patients with concomitant NSAID use. This novel association was not diminished after adjusting for potential confounders, and is an intriguing area for future investigation as the impact of NSAIDs on eosinophils has been little studied. ${ }^{31}$ It is biologically plausible that NSAIDs aid in reducing the esophageal inflammation by inhibiting COX-2 enzyme production of prostaglandins and other inflammatory mediators. This mechanism has been studied in other esophageal diseases, but has yet to be explored in EoE. ${ }^{17,32}$ It is also possible to speculate that NSAIDs, if used chronically, might help to desensitize from allergic conditions, as they do in aspirin-exacerbated respiratory disease. ${ }^{33}$ Due to potential side effects, more investigation of mechanism, dose, frequency, and specific medication would be essential before NSAIDs could be considered as an adjunctive therapy in EoE.

There are several limitations of this study. First, this was a single-center study at a referral institution, which could impact generalizability of the results. However, the enrolled EoE population has similar characteristics to those reported at other centers, and the requirement to enroll only incident (and therefore treatment naïve) EoE cases helps to counter this limitation. Second, exposure data were self-reported and we lacked data on dose, frequency, and timing of NSAID use, which was not included when the original questionnaire was created. Therefore, we were not able to perform dose-response analyses or explore the effects of different NSAIDs. There is no reason to suspect that exposure reporting would be differential between cases and controls, particularly because exposure data were collected prior to the known diagnosis of EoE. Additionally, misclassification of exposures would likely bias toward the null. Partial exposure data was missing for some of the patients but the size of the study population minimizes the potential effect on the results. Follow-up to assess treatment response was available on only 86 of our 115 patients. We also used an endoscopic determination of fibrostenosis, but it has been noted that endoscopy may not be as sensitive as barium esophagram for findings of esophageal narrowing. ${ }^{34}$ While it is a potential limitation that our control group was not population- based and included GERD patients in whom smoking and alcohol could be though to be more common, it is notable that the controls with GERD had similar rates of smoking than controls with non-GERD symptoms or diagnoses, and that alcohol use was actually more common in EoE patients on unadjusted analysis. In addition, by focusing on patients undergoing upper endoscopy with upper GI symptoms, the non-EoE controls were felt to represent the sampling base for the cases. We also note that with this case-control study design, we can only report associations and not causal relationships.

The study design also had several strengths. To date, this was the largest and only prospective study on this topic. The study was rigorously conducted, with uniform data collection methods throughout. The control group was designed to represent the source population of patients presenting with suspected EoE and was not simply reflective of a GERD population, so would be pertinent for risk factor evaluation in patients coming to endoscopic evaluation. In addition, we were able to assess treatment response via follow up data on a large subset of the EoE cases.

In conclusion, we found that smoking and NSAIDs were inversely associated with EoE as compared to endoscopy-based non-EoE controls, and that this relationship persisted after multivariate analysis. Alcohol consumption was more common among EoE patients but was not an independent risk factor after controlling for confounders. While none of the exposures were associated with a fibrostenotic phenotype, these data suggested that NSAID use may be associated with histologic response to treatment. Further study of NSAIDs as a possible adjunctive therapy for EoE should be considered.

\section{SUPPLEMENTARY DATA}

Supplementary data are available at DOTESO online.

\section{ACKNOWLEDGEMENT}

Financial support: This work was supported, in part, by NIH Awards T35DK007386 (NTK), K23DK090073 (ESD), K24DK100548 (NJS), and T32DK007634 (SE), and uses resources from the UNC Center for GI Biology and Disease (P30DK34987).

\section{References}

1 Liacouras C A, Furuta G T, Hirano I et al. Eosinophilic esophagitis: updated consensus recommendations for children and adults. J Allergy Clin Immunol 2011; 128: 3-20.e6.

2 Dellon E S, Gonsalves N, Hirano I, Furuta G T, Liacouras C, Katzka D A. ACG Clinical Guideline: evidence based approach to the diagnosis and management of esophageal eosinophilia 
and eosinophilic esophagitis. Am J Gastroenterol 2013; 108 679-92.

3 Furuta G T, Katzka D A. Eosinophilic esophagitis. N Engl J Med 2015; 373: 1640-8.

4 Jensen E T, Kappelman M D, Martin C F, Dellon E S. Healthcare utilization, costs, and the burden of disease related to eosinophilic esophagitis in the United States. Am J Gastroenterol 2015; 110: 626-32.

5 Desai T K, Stecevic V, Chang C H, Goldstein N S, Badizadegan K, Furuta G T. Association of eosinophilic inflammation with esophageal food impaction in adults. Gastrointest Endosc 2005; 61: 795-801.

6 Kerlin P, Jones D, Remedios M, Campbell C. Prevalence of eosinophilic esophagitis in adults with food bolus obstruction of the esophagus. J Clin Gastroenterol 2007; 41: 356-61.

7 Sperry S L, Crockett S D, Miller C B, Shaheen N J, Dellon E S. Esophageal foreign-body impactions: epidemiology, time trends, and the impact of the increasing prevalence of eosinophilic esophagitis. Gastrointest Endosc 2011; 74: 985-91.

8 Heine R G. Insights into the emerging epidemic of eosinophilic oesophagitis. Best Pract Res Clin Gastroenterol 2015; 29: 731-7.

9 Dellon E S, Jensen E T, Martin C F, Shaheen N J, Kappelman M D. Prevalence of eosinophilic esophagitis in the United States. Clin Gastroenterol Hepatol 2014; 12: 589-96.e1.

10 Dellon E S. Epidemiology of eosinophilic esophagitis. Gastroenterol Clin North Am 2014; 43: 201-18.

11 Lipka S, Kumar A, Richter J E. Impact of Diagnostic delay and other risk factors on eosinophilic esophagitis phenotype and esophageal diameter. J Clin Gastroenterol 2016; 50: 134-40.

12 Martin-de-Argila C, Martinez-Jimenez P. Epidemiological study on the incidence of gastroesophageal reflux disease symptoms in patients in acute treatment with NSAIDs. Expert Rev Gastroenterol Hepatol 2013; 7: 27-33.

13 Ishiguro S, Sasazuki S, Inoue M, Kurahashi N, Iwasaki M, Tsugane S. Effect of alcohol consumption, cigarette smoking and flushing response on esophageal cancer risk: a populationbased cohort study (JPHC study). Cancer Lett 2009; 275: 240-6.

14 Lagergren J, Bergstrom R, Lindgren A, Nyren O. The role of tobacco, snuff and alcohol use in the aetiology of cancer of the oesophagus and gastric cardia. Int J Cancer 2000; 85: 340-6.

15 Chiba H, Gunji T, Sato H et al. A cross-sectional study on the risk factors for erosive esophagitis in young adults. Intern Med 2012; 51: 1293-9.

16 Omer Z B, Ananthakrishnan A N, Nattinger K J et al. Aspirin protects against Barrett's esophagus in a multivariate logistic regression analysis. Clin Gastroenterol Hepatol 2012; 10: 722 7.

17 Corley D A, Kerlikowske K, Verma R, Buffler P. Protective association of aspirin/NSAIDs and esophageal cancer: a systematic review and meta-analysis. Gastroenterology 2003; 124: $47-56$.

18 Dellon E S, Rusin S, Gebhart J H et al. Utility of a noninvasive serum biomarker panel for diagnosis and monitoring of EoE: a prospective study. Am J Gastroenterol 2015; 110: $821-7$.
19 Dellon E S, Rusin S, Gebhart J H et al. A clinical prediction tool identifies cases of eosinophilic esophagitis without endoscopic biopsy: a prospective study. Am J Gastroenterol 2015; 110: $1347-54$

20 Dellon E S, Gebhart J H, Higgins LL, Hathorn K E, Woosley J T, Shaheen N J. The esophageal biopsy "pull" sign: a highly specific and treatment-responsive endoscopic finding in eosinophilic esophagitis (with video). Gastrointest Endosc 2016; 83: 92-100.

21 Dellon E S, Cotton C C, Gebhart J H et al. Accuracy of the eosinophilic esophagitis endoscopic reference score in diagnosis and determining response to treatment. Clin Gastroenterol Hepatol 2016; 14: 31-9.

22 Dellon E S, Fritchie K J, Rubinas T C, Woosley J T, Shaheen $\mathrm{N} \mathrm{J}$. Inter- and intraobserver reliability and validation of a new method for determination of eosinophil counts in patients with esophageal eosinophilia. Dig Dis Sci 2010; 55: 1940-9.

23 Rusin S, Covey S, Perjar I et al. Determination of esophageal eosinophil counts and other histologic features of eosinophilic esophagitis by pathology trainees is highly accurate. Hum Pathol 2017; 62: 50-5.

24 Dellon E S, Aderoju A, Woosley J T, Sandler R S, Shaheen N J. Variability in diagnostic criteria for eosinophilic esophagitis: a systematic review. Am J Gastroenterol 2007; 102: 2300-13.

25 Dellon E S, Liacouras C A. Advances in clinical management of eosinophilic esophagitis. Gastroenterology 2014; 147: 1238-54.

26 Wolf W A, Cotton C C, Green D J et al. Evaluation of histologic cutpoints for treatment response in eosinophilic esophagitis. J Gastroenterol Hepatol Res 2015; 4: 1780-7.

27 Runge T M, Dellon E S. Do we know what causes eosinophilic esophagitis? A mechanistic update. Curr Gastroenterol Rep 2015; 17: 458

28 Sherrill J D, Kc K, Wu D et al. Desmoglein-1 regulates esophageal epithelial barrier function and immune responses in eosinophilic esophagitis. Mucosal Immunol 2014; 7: 718-29.

29 Katzka D A, Ravi K, Geno D M et al. Endoscopic mucosal impedance measurements correlate with eosinophilia and dilation of intercellular spaces in patients with eosinophilic esophagitis. Clin Gastroenterol Hepatol 2015; 13: 1242-8 e1.

30 van Rhijn B D, Weijenborg P W, Verheij J et al. Proton pump inhibitors partially restore mucosal integrity in patients with proton pump inhibitor-responsive esophageal eosinophilia but not eosinophilic esophagitis. Clin Gastroenterol Hepatol 2014; 12: $1815-23$ e2.

31 Fulkerson P C, Rothenberg M E. Targeting eosinophils in allergy, inflammation and beyond. Nat Rev Drug Discovery 2013; 12: 117-29.

32 Thrift A P, Anderson L A, Murray L J et al. Nonsteroidal antiinflammatory drug use is not associated with reduced risk of Barrett's esophagus. Am J Gastroenterol 2016; 111: 1528-35.

33 Hill J, Burnett T, Katial R. Mechanisms of benefit with aspirin therapy in aspirin-exacerbated respiratory disease. Immunol Allergy Clin North Am 2016; 36: 735-47.

34 Gentile N, Katzka D, Ravi K et al. Oesophageal narrowing is common and frequently under-appreciated at endoscopy in patients with oesophageal eosinophilia. Aliment Pharmacol Ther 2014; 40: 1333-40. 